\title{
Quality improvement education to improve performance on ulcerative colitis quality measures and care processes aligned with National Quality Strategy priorities
}

\author{
Laurence Greene, Kathleen Moreo \\ PRIME Education, Inc. (PRIME®), USA
}

\begin{abstract}
Studies on inflammatory bowel disease (IBD) have reported suboptimal approaches to patient care. In the United States, the findings have motivated leading gastroenterology organizations to call for initiatives that support clinicians in aligning their practices with quality measures for IBD and priorities of the National Quality Strategy (NQS).

We designed and implemented a quality improvement (QI) education program on ulcerative colitis in which patient charts were audited for 30 gastroenterologists before ( $n=300$ charts) and after $(n=290$ charts) they participated in Ql-focused educational activities. Charts were audited for nine measures, selected for their alignment with four NQS priorities: making care safer, ensuring patient engagement, promoting communication, and promoting effective treatment practices. Four of the measures, including guideline-directed vaccinations and assessments of disease type and activity, were part of the CMS Physician Quality Reporting System (PQRS). The other five measures involved counseling patients on various topics in ulcerative colitis management, documentation of side effects, assessment of adherence status, and simplification of dosing. The gastroenterologists also completed baseline and post-education surveys designed to assess qualitative outcomes.
\end{abstract}

One of the educational interventions was a private audit feedback session conducted for each gastroenterologist. The sessions were designed to support participants in identifying measures reflecting suboptimal care quality and developing action plans for improvement. In continuous improvement cycles, follow-up interventions included QI tools and educational monographs.

Across the nine chart variables, post-education improvements ranged from $0 \%$ to $48 \%$, with a mean improvement of $15.9 \%$. Survey findings revealed improvements in self-reported understanding of quality measures and intentions to apply them to practice, and lower rates of perceived significant barriers to high-quality care. The findings indicate the potential for QI education to support gastroenterologists in improving their performance on key measures of care quality for patients with ulcerative colitis.

\section{Problem}

This quality improvement (QI) program and outcomes evaluation was conducted by PRIME Education, Inc. (PRIME®), a national medical education company and multi-accredited provider of continuing education for the health care team. Through literature reviews and interviews with national leaders in QI initiatives in the field of inflammatory bowel disease (IBD), we identified needs for education among U.S. community-based gastroenterologists to address published reports of deficiencies and variability in the quality of care provided to patients with ulcerative colitis. Along with Crohn's disease, ulcerative colitis is a type of inflammatory bowel disease (IBD). As described in the following section, studies have reported suboptimal physician adherence to recommended IBD quality of care processes and patient counseling to ensure safe and effective treatment outcomes. Historically, U.S. undergraduate and graduate medical education programs have not provided training in QI principles and methods.

In the context of U.S. health care reform, the need for Ql-focused continuing education among gastroenterologists is compounded by recently instated requirements for performing, documenting, and reporting IBD quality measures for reimbursement and to avoid payment penalties. These requirements are enforced in programs such as the Centers for Medicare and Medicaid Services (CMS) Physician Quality Reporting System (PQRS), in which physician performance is assessed through clinical, process-based quality measures and patient-centered measures that include provision of counseling, education, and shared decision-making. Many patientcentered quality measures align with priorities of the National Quality Strategy (NQS), which include making care safer, ensuring that patients are engaged as partners in their care, promoting effective communication with patients, and promoting effective treatment practices. Among reported barriers to successful performance on quality measures are physicians' lack of knowledge about their evidence-based rationale, ineffective documenting strategies, and concerns about administrative burdens, costs, and time commitments.

\section{Background}

Over the past decade, a number of studies in North America have reported suboptimal and variable quality of care for patients with inflammatory bowel disease (IBD). For example, evidence from 
studies conducted in the United States indicates that low percentages of patients with ulcerative colitis or Crohn's disease receive recommended influenza and pneumococcal vaccinations.[1-3] In one study, nearly $50 \%$ of IBD patients reported that their physicians had not informed them of the need for the vaccinations.[1] Suboptimal rates of guideline-directed screening for tuberculosis and hepatitis $B$ virus have been reported in patients with IBD before initiation of immunosuppressive therapy.[4] In addition, across different patient subpopulations and regions of the country, studies have indicated significant disparity in the quality of IBD care.[5,6] In a survey study, only $10 \%$ to $36 \%$ of patients with IBD reported receiving the right amount of information about the disease symptoms, possible complications, long-term prognosis, medication side effects, and self-management of symptoms.[7] Moreover, only $8 \%$ to $16 \%$ of the patients reported receiving the correct amount of information about lifestyle modifications including dietary changes.[7]

Experts in the field of QI have recognized the potential for continuing education programs to improve physicians' performance on quality measures and to thereby enhance patient outcomes.[8] Preliminary studies have demonstrated this potential in areas including diabetes, venous thromboembolism, and radiation oncology.[9-11] We recently reported a study showing a positive impact of QI education on chart-documented rates of adherence to Physician Quality Reporting System (PQRS) quality measures for IBD among a group of low-performing gastroenterologists in their treatment of patients with Crohn's disease.[12] The study's main educational intervention was chart audit feedback.

This article and the results of our program are designed to support community-based gastroenterologists in improving performance on quality of care processes related to National Quality Strategy (NQS) priorities for patients with ulcerative colitis.

\section{Baseline measurement}

Through a systematic process to identify community-based gastroenterology practices with large numbers of ulcerative colitis patients, we recruited 30 gastroenterologists in 16 states to participate in the QI program's chart audits and educational activities. Clinical staff from each practice were also invited to participate. At baseline, the administrative staff in each practice randomly selected an oversample of up to 15 charts of patients who met the following inclusion criteria: aged 18 years or older, diagnosis of mild or moderate ulcerative colitis in the 24 month period of April 2011 to April 2013, and least one visit with the provider in the 12 month period of April 2012 to April 2013. Trained medical record reviewers retrospectively audited a total of 300 baseline charts; the review period was one year, from April 1, 2012 and April 1, 2013. Charts were audited for patient demographics and disease characteristics, and documentation for nine measures, which were selected for their alignment with key aspects of four of the six National Quality Strategy (NQS) priorities: making care safer by reducing harm caused in the delivery of care, ensuring that patients are engaged as partners in their care, promoting effective communication, and promoting effective treatment practices.[13]
For each participant, we calculated percentages of baseline charts that documented physician performance or patient outcome for each of the nine measures. Overall baseline values were then calculated as the means for each measure across the 30 gastroenterologists. Baseline percentages are listed as follows (also see table 1 in the supplementary file). Note that the first eight measures are physician performance measures for which lower percentages reflect suboptimal quality of care. For the ninth measure, nonadherence of patients to their medications, a lower percentage reflects a better patient outcome. The first four measures are evidence-based quality measures in the PQRS program for IBD. These measures are related to NQS priorities for making care safer and promoting effective treatment practices. Measures five and six are related to the NQS priorities for engaging patients as partners in their care and promoting effective communication. Measures seven to nine are related to NQS priorities for making care safer and promoting effective treatment practices.

1. Influenza immunization: $4 \%$

2. Pneumococcal immunization: $0.3 \%$

3. Assessment of disease type: $35 \%$

4. Assessment of disease activity: $13 \%$

5. Counseling for medication risks/benefits and adherence: $32 \%$

6. Counseling for lifestyle modifications: $15 \%$

7. Documentation of side effects: $4 \%$

8. Simplification of ulcerative colitis therapy dosing : $56 \%$

9. Nonadherence to medications: $30 \%$

Across the first eight measures, the mean was $19.9 \%$, which indicates relatively low rates of baseline performance. The relatively high rate of patient non-adherence to medications also reflects an area for quality improvement.

In addition to participating in the chart audits, the 30 gastroenterologists completed baseline and post-education surveys designed to assess self-reported understanding of quality measures, intentions to apply quality measures to practice, selfreported ability to make evidence-based treatment decisions, and barriers to providing high-quality ulcerative colitis care. Baseline survey results are included in table 2 in the supplementary file.

\section{Design}

A primary educational intervention was a live one hour private audit feedback session conducted for each gastroenterologist and his/her clinical staff, held within one month after the practice's baseline chart audits. These sessions were led by a clinician with experience in presenting chart audit data and an expert gastroenterologist involved in national IBD quality improvement initiatives. During a session, the participating gastroenterologist's baseline measures were presented and compared with pooled, de-identified measures from the entire cohort. In cases when a physician's performance met high standards (eg, exceeding an adherence rate of $80 \%$ or surpassing the cohort's mean adherence rate), the expert gastroenterologist gave feedback consistent with positive reinforcement. When the performance of a measure was 
substandard, the expert addressed the evidence-based rationale for the measure, engaged the participant in discussing barriers to achieving high levels of performance, and collaborated with the participant in developing an action plan for improvement.

The initial design of the audit feedback sessions was informed by reviews of published studies and meta-analyses in the area of continuing education for health care professionals. This educational method has generally been associated with small but statistically and clinically significant improvements in health care practice.[14,15] Analyses indicate that the greatest improvements occur when feedback is provided more than once, offered by a supervisor or colleague, and accompanied by specific goals or action plans for quality improvement.[14] In addition, the effects of audit feedback education are greatest for health care professionals with low baseline performance scores.[12,14]

To reinforce QI education themes from the audit feedback sessions, we developed two online- and mobile-accessible evidence-based monographs as additional educational interventions. One of the monographs presented principles, tools, and strategies for improving the quality of care and outcomes for patients with ulcerative colitis. The other monograph covered evidence-based approaches to the safe and effective medical management of the disease. For these follow-up activities, we chose monographs because our needs assessments have indicated that physicians value evidence-based literature that is accessible for repeated referral, to continually reinforce concepts presented in in-person educational activity formats.

\section{Strategy}

Improvement cycles were based on feedback that we received from the participating gastroenterologists through their baseline surveys and communication during and after the audit feedback sessions. This input informed revisions of the educational content and processes of subsequent audit feedback sessions. The input also directed our provision of tools and resources to address gaps and needs for individual physicians as well as the entire cohort. For example, in the initial audit feedback activities, several participants asked for guidance and tools for improving their performance and documentation of PQRS quality measures for IBD and their adherence to evidence-based clinical recommendations. In response to this input, we developed downloadable QI tools and subsequently reached out to the gastroenterologists and their clinical teams at least three times each to encourage access and utility of the tools. We also established a method for the gastroenterologists to obtain the QI education tools and applicable resources in online- and mobile-accessible formats from our QIfocused website at www.CMEToolkit.com. This intervention was successful, with over $70 \%$ of the gastroenterologists accessing the website. Access to the tools was obtained through mobile and online devices.

With a goal to reach all of the gastroenterologists, our next QI intervention cycle was more direct. We asked the expert gastroenterologists who led the audit feedback sessions to follow up with participants by email to share their own QI tools, including checklists for performing and documenting key quality measures. We followed up with the gastroenterology offices to assess whether and when the tools were received and utilized by the clinical team members. This peer-to-peer exchange was particularly well received by the participating gastroenterologists.

Our next intervention was to provide online- and mobile-accessible educational monographs to the participating gastroenterologists and their clinical teams. Two multi-accredited monographs were added to the online QI toolkit and were adapted to reinforce key themes for improving performance on NQS-related quality of care processes. Through our continuous telephonic concierge service we tracked the access to and downloads of the independently accredited monographs, as well as gathered pre-education and post-education surveys to measure learners' increases in knowledge, confidence, and competence resulting from participation in the accredited monograph activities.

Our final and perhaps most effective improvement cycle involved providing each gastroenterology practice with an analysis of its baseline and post-education measures. Six months after all gastroenterologists completed their improvement cycles and all follow-up chart audits were completed, we compiled and analyzed the data across the practices. We then provided an individualized report to each gastroenterology practice, which allowed each gastroenterologist and his/her clinical team to assess their improvement in adherence to the NQS-related measures, compared with the 29 other practices participating in the program.

\section{Results}

Six months after each gastroenterologist in the cohort completed the educational activities, administrative staff in their practices randomly selected an oversample of up to 15 patient charts according to the same method used for the baseline chart selection. To be included, patients must have had at least one visit with the physician in the six months after the education. A total of 290 randomly selected charts were retrospectively audited for patient demographics, disease characteristics, and the same nine measures from the baseline audits. One physician dropped out of the program for personal reasons after the educational interventions.

Baseline and post-education results of the chart audits are presented in table 1 in the supplementary file. Improvements were observed for the following measures:

- Influenza vaccination (+21\%) and pneumococcal vaccination (+5.7 $\%)$

- Provision of patient counseling about medication risks/benefits and adherence (+48\%) and lifestyle modifications (+23\%)

- Nonadherence to medications (-24\%; negative percentages indicate improvement from baseline)

- Simplification of ulcerative colitis therapy dosing $(+14 \%)$ 
Minimal or no changes were observed for documentation of disease type $(0 \%)$, disease severity $(+3 \%)$, and side effects $(+4 \%)$.

As shown in table 2 in the supplementary file, the survey results indicated substantial post-education improvements in participants' self-reported "excellent" understanding of quality measures $(+37 \%)$, intentions to apply quality measures to practice $(+37 \%)$, selfreported "excellent" ability to make evidence-based treatment and management decisions $(+16 \%)$, and intention to simplify ulcerative colitis treatment regimens before escalating therapies $(+50 \%)$. In addition, there were reductions in the numbers of physicians who viewed the following barriers as "very significant" in the management of patients with ulcerative colitis: side effects of medications $(-17 \%)$, lack of familiarity with new treatments $(-10 \%)$, and lack of familiarity with practice guidelines (-16\%).

See supplementary file: ds5307.docx - "Supplementary Tables"

\section{Lessons and limitations}

Through this project, we have learned that the effective integration of continuing education into QI programs must account for a number of potential barriers. For example, some of the gastroenterologists who participated in the chart audits pointed out that the lack of explicit chart documentation does not necessarily indicate poor quality care in relation to NQS priorities. Moreover, some participants questioned whether adherence to process-based quality of care measures translates into significantly improved patient outcomes. As we learned about these attitudinal barriers in early phases of the educational program, we adapted our interventions accordingly. For example, the expert gastroenterology faculty addressed gaps in regular, quality-focused chart documentation by engaging participants in discussion about implications for suboptimal care and medical errors that compromise patient safety. In addition, we added QI reporting tools to the online toolkit.

Our observations of peer-to-peer communication during the audit feedback sessions revealed a number of interesting themes regarding participants' attitudes and perceptions about challenges of improving performance on quality measures for ulcerative colitis and NQS-related care processes. Through observing peer-to-peer communications during the in-person educational activities, we gained insights into the participating physicians' reasons for not always adhering to quality measures. A key reason was lack of time during patient visits. In addition, the gastroenterologists cited barriers involving care coordination with primary care physicians. As an example, several gastroenterologists said that their patients should receive influenza and pneumococcal immunizations from their primary care physicians. However, in some cases these gastroenterologists had no documentation of whether these services were actually provided. The lesson learned is that QI education must directly address gaps in care coordination and health system fragmentation, extending beyond the cohort or organization involved in a QI project.

This program lacked educational interventions for patients, which may have influenced the extent to which improvements were observed in the follow-up chart audits. Other challenges involved the logistics of carrying out the project. Gastroenterologists are busy, with limited time for education, requiring us to find creative ways to keep them engaged in the program. For example, through continuous email and phone communication, we updated participants about the program and reminded them to complete educational activities and surveys. We provided educational materials and study surveys in various forms (eg, online, mobileaccessible, and Express Mail print copies) to facilitate application. To manage the logistical challenges in successfully implementing a QI education project, the various members of the education provider team must operate in a continuous improvement model, with effective interprofessional collaboration and communication.

Through this project, we learned that delivering several cycles of accredited education can be an effective way of keeping clinicians, especially busy specialty physicians, engaged in QI initiatives. The accredited activities provide a recognized and respected incentive for the specialists and deliver important information about QI methods in ways that address clinicians' learning needs and habits.

\section{Conclusion}

We observed small to substantial post-education increases in rates of performance of national quality measures for IBD and other care processes aligned with NQS priorities. In addition, the survey data reflected improvements in self-reported understanding and intentions to change practice for better patient outcomes, as well as more positive perceptions regarding barriers to the provision of highquality ulcerative colitis care. The results of this project indicate the potential for QI education to support community-based gastroenterologists in improving their performance of clinical skills that relate to priorities of the U.S. National Quality Strategy. The project results and lessons learned offer insights into designing future programs with educational interventions that address barriers to quality improvement in the management of patients with ulcerative colitis.

\section{References}

1. Melmed GY, Ippoliti AF, Papadakis KA, et al. Patients with inflammatory bowel disease are at risk for vaccinepreventable illnesses. Am J Gastroenterol 2006;101(8):1834-40.

2. Wasan SK, Coukos JA, Farraye FA. Vaccinating the inflammatory bowel disease patient: deficiencies in gastroenterologists knowledge. Inflamm Bowel Dis 2011;17(12):2536-40.

3. Yeung JH, Goodman KJ, Fedorak RN. Inadequate knowledge of immunization guidelines: a missed opportunity for preventing infection in immunocompromised IBD patients. Inflamm Bowel Dis 2012;18(1):34-40.

4. Wasan SK, Calderwood AH, Long MD, Kappelman MD, Sandler RS, Farraye FA. Immunization rates and vaccine beliefs among patients with inflammatory bowel disease: an opportunity for improvement. Inflamm Bowel Dis 2014;20(2):246-50. 


\section{BMJ Quality Improvement Reports}

5. Nguyen GC, Laveist TA, Gearhart S, Bayless TM, Brant SR. Racial and geographic variations in colectomy rates among hospitalized ulcerative colitis patients. Clin Gastroenterol Hepatol 2006;4(12):1507-13.

6. Kappelman MD, Palmer L, Boyle BM, Rubin DT. Quality of care in inflammatory bowel disease: a review and discussion. Inflamm Bowel Dis 2010;16(1):125-33.

7. Wong S, Walker JR, Carr R, Graff LA, Clara I, Promislow S, et al. The information needs and preferences of persons with longstanding inflammatory bowel disease. Can J Gastroenterol 2012 Aug;26(8):525-31.

8. Shojania KG, Silver I, Levinson W. Continuing medical education and quality improvement: a match made in heaven? Ann Intern Med 2012;156(4):305-8.

9. Peterson LE, Blackburn B, Phillips RL, Puffer JC. Improving quality of care for diabetes through a maintenance of certification activity: family physicians' use of the chronic care model. J Contin Educ Health Prof 2014;34(1):47-55

10. Al-Hameed F, Al-Dorzi HM, Aboelnazer E. The effect of a continuing medical education program on venous thromboembolism prophylaxis utilization and mortality in a tertiary-care hospital. Thromb J 2014;12:9.

11. Leong CN, Shakespeare TP, Mukherjee RK, et al. Efficacy of an integrated continuing medical education (CME) and quality improvement (QI) program on radiation oncologist (RO) clinical practice. Int J Radiat Oncol Biol Phys 2006;66(5):1457-60.

12. Greene L, Sapir T, Moreo K, Carter J, Higgins PD. Impact of quality improvement education on documented adherence to quality measures for adults with Crohn's disease. Inflamm Bowel Dis (in press).

13. Agency for Healthcare Research and Quality (AHRQ). The National Quality Strategy (NQS). http://www.ahrq.gov/workingforquality/.

14. Ivers N, Jamtvedt G, Flottorp S, et al. Audit and feedback: effects on professional practice and healthcare outcomes. Cochrane Database Syst Rev 2012;6:CD000259.

15. Bloom BS. Effects of continuing medical education on improving physician clinical care and patient health: a review of systematic reviews. Int J Technol Assess Health Care 2005;21(3):380-5.

\section{Declaration of interests}

The authors represent PRIME Education, Inc, a health care education company that received an independent educational grant from Shire to conduct the quality improvement project described in this article. Shire had no role in the study design or execution, and the grant did not include support for writing this manuscript.

\section{Acknowledgements}

Tamar Sapir, PhD; Jeffrey Carter, PhD; Barry Patel, PharmD.

\section{Ethical approval}

Independent institutional review board approval was granted before participants were recruited and enrolled in this quality improvement education program (Sterling IRB, Atlanta, GA, USA; IRB ID 4290). 